\title{
3D Object Recognition Using X3D and Deep Learning
}

\author{
HA-SEONG KIM \\ The University of Suwon, Hwaseong, Gyeonggi-do, 18323, \\ Republic of Korea
}

\begin{abstract}
In this paper, a method of recognizing a 3D object using a machine learning algorithm is described. 3D object data sets consisting of geometric polygons are analyzed by Keras, a deep learning API that learns composition rules of data sets. A 3D object can be recognized by applying a composition rule to the object. Data sets for various types of objects have been experimented with. 100 objects per shape were used to learn the rules and different objects were used for evaluating the rules. Seven types of 3D objects were experimented with. In addition, evaluation results for different numbers of 3D objects were compared.
\end{abstract}

\section{CCS CONCEPTS}

- Computer graphics; • Graphics systems and interface; • Perception;

\section{KEYWORDS}

3D object recognition, 3D machine learning, 3D deep learning, sequential model, 3D training data, 3D building model, training model

\section{ACM Reference Format:}

HA-SEONG KIM and MYEONG WON LEE. 2020. 3D Object Recognition Using X3D and Deep Learning. In The 25th International Conference on 3D Web Technology (Web3D '20), November 09-13, 2020, Virtual Event, Republic of Korea. ACM, New York, NY, USA, 8 pages. https://doi.org/10.1145/3424616. 3424703

\section{INTRODUCTION}

3D graphics objects are increasingly used in various areas and this often includes a large set of 3D objects. In order to manipulate 3D objects, a method of retrieving them is needed. This paper describes a method of recognizing a 3D object using machine learning technology [Shanahan and Dai 2019]. Much research has been done on object recognition, but most of this has involved detecting an object using 2D pixel data obtained from camera generated images. In this case, objects are recognized by analyzing pixel data [Hong et al. 2019; Zhou et al. 2017; He et al. 2015]. Compared to $2 \mathrm{D}$ object recognition in image data, $3 \mathrm{D}$ object recognition requires the analysis of 3D data for an object. In research, a 3D scene is typically represented as discrete grids and then converted to volumetric data [Peng et al. 2015]. Although some research is related

Permission to make digital or hard copies of all or part of this work for personal or classroom use is granted without fee provided that copies are not made or distributed for profit or commercial advantage and that copies bear this notice and the full citation on the first page. Copyrights for components of this work owned by others than ACM must be honored. Abstracting with credit is permitted. To copy otherwise, or republish, to post on servers or to redistribute to lists, requires prior specific permission and/or a fee. Request permissions from permissions@acm.org.

Web3D '20, November 09-13, 2020, Virtual Event, Republic of Korea

(c) 2020 Association for Computing Machinery.

ACM ISBN 978-1-4503-8169-7/20/11 . \$ $\$ 15.00$

https://doi.org/10.1145/3424616.3424703

\author{
MYEONG WON LEE \\ The University of Suwon, Hwaseong, Gyeongg-do, 18323, \\ Republic of Korea
}

to object recognition using 3D models, image recognition methods are usually used by expanding one more dimension over and above that of conventional 2D object recognition [Zhi et al. 2017; Zhang et al. 2017]. Compared to image-based methods, this paper uses the geometric coordinates of a composed 3D object [Rodrigues and Robinson 2011]. This method has the advantages of more accurate $3 \mathrm{D}$ data analysis versus previous image detection methods because geometric coordinates are used directly [Berretti et al. 2018].

\section{MACHINE LEARNING ALGORITHM FOR 3D OBJECT RECOGNITION}

\subsection{Model / Layer Hierarchy}

There are several machine learning algorithms such as CNN (Convolution Neural Network), DNN (Deep Neural Network, and RNN (Recurrent Neural Network). CNN is a deep learning algorithm used to classify image, video, text, and sound. This is useful when searching for patterns for recognizing an object or a person in an image. DNN is a model that includes multiple hidden layers between input and output layers. This is useful for categorizing and estimating data. RNN is a class of artificial neural networks where connections between nodes form a directed graph along a temporal sequence.

In this paper, the DNN model algorithm was used since a 3D object should be recognized by estimating what shape it is based on a sequence of input 3D values. 3D vector data for an object corresponds to the input values and therefore a sequential model was used for machine learning.

\subsection{Loss Function}

A loss function, also called a cost function, is used to evaluate how much the training data using a machine and the estimated data differ. There are several loss functions used for machine learning algorithms such as MSE (Mean Squared Error) and CE (Cross Entrophy) [Zhang and Sabuncu 2018]. In this paper, CCEL (Categorical Cross Entrophy Loss) was used because categorization of 3D geometric data is more appropriate than using linear regression for machine learning [Ho and Wookey 2010].

\subsection{Optimizer}

An optimizer is intended to search a parameter value to minimize loss functions due to neural network learning and its results [Rudger 2017]. The stochastic gradient descent (SDG) is used to obtain an optimal weighted value through updating weighted values by gradient with a differential. In this paper, the Adam optimizer (Adaptive Moment estimation) [Kingma 2015], an algorithm combining Momentum and RMSProp [Mukkamala and Hein 2017], was used to obtain a minimum value for a loss function. The value is obtained by applying average exponent movement and by an adaptive vector for obtaining direction and size of variables. 


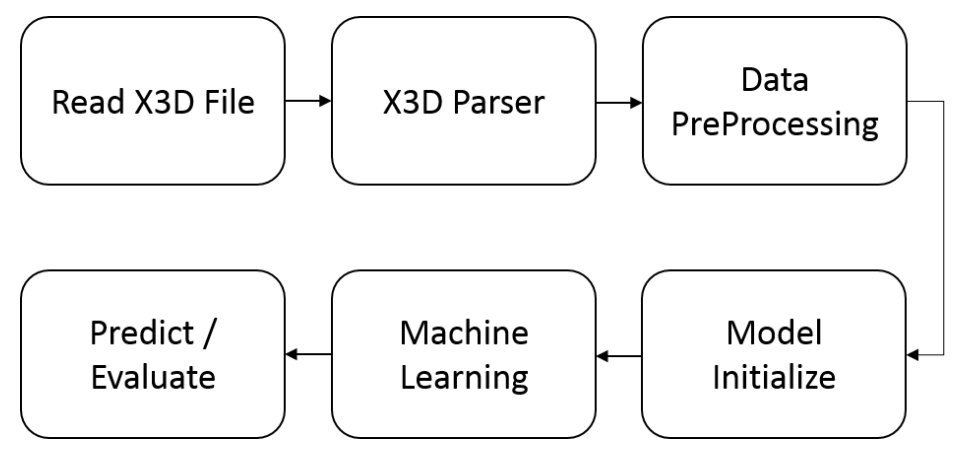

Figure 1: Procedure of 3D object recognition using machine learning

\subsection{Library}

In this paper, Keras, an open source library written by Python and operated in Tensorflow, was used for machine learning. 3D data sets of several primitives and specific 3D models are input for training using the Keras Deep Learning API and the rule model of organizing the $3 \mathrm{D}$ data sets is obtained. Then, a new object is evaluated and estimated for the trained model with recognition of how much the object is similar to the trained model.

\section{A 3D OBJECT RECOGNITION ALGORITHM}

The 3D object recognition algorithm consists of six steps for processing 3D objects (Figure 1). For 3D objects, the X3D standard was used in this paper [ISO/IEC 2013]. First, a 3D data set is read.
Second, 3D files are parsed to obtain the necessary information. Third, data preprocessing is done to match data formats for machine learning. Fourth, a model for training is defined, which is model initialization. Fifth, the 3D data set is trained using the model for machine learning. The final step is to predict and evaluate the object using the trained model.

\subsection{Read X3D File}

In order to process machine learning, data mining is necessary to discover patterns in large data sets. Data mining extracts information from a data set and transforms the information into a comprehensible structure using statistical regularities or patterns. For this process, sets of 3D primitives or objects stored as X3D files

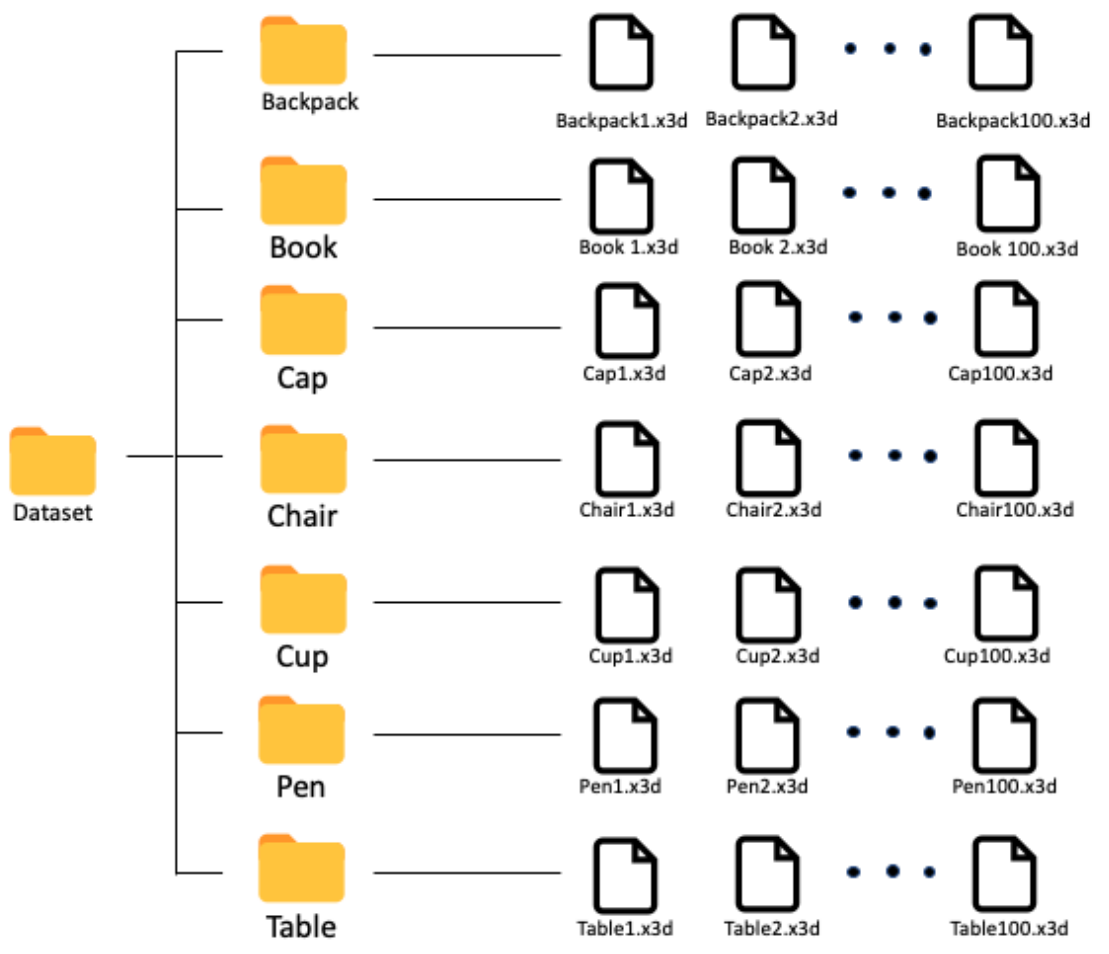

Figure 2: A structure of directories for a data set to be tested for machine learning 


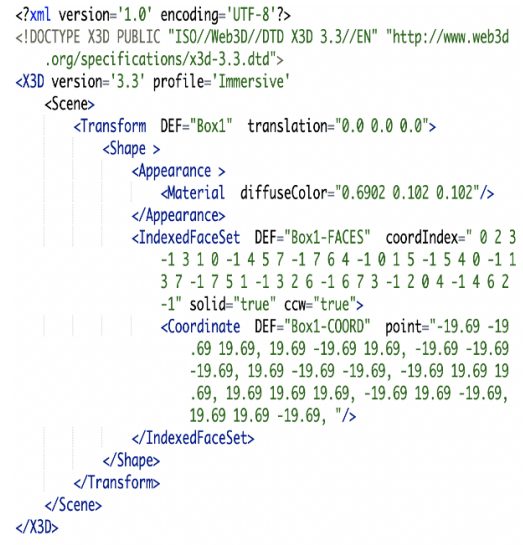

X3D Source

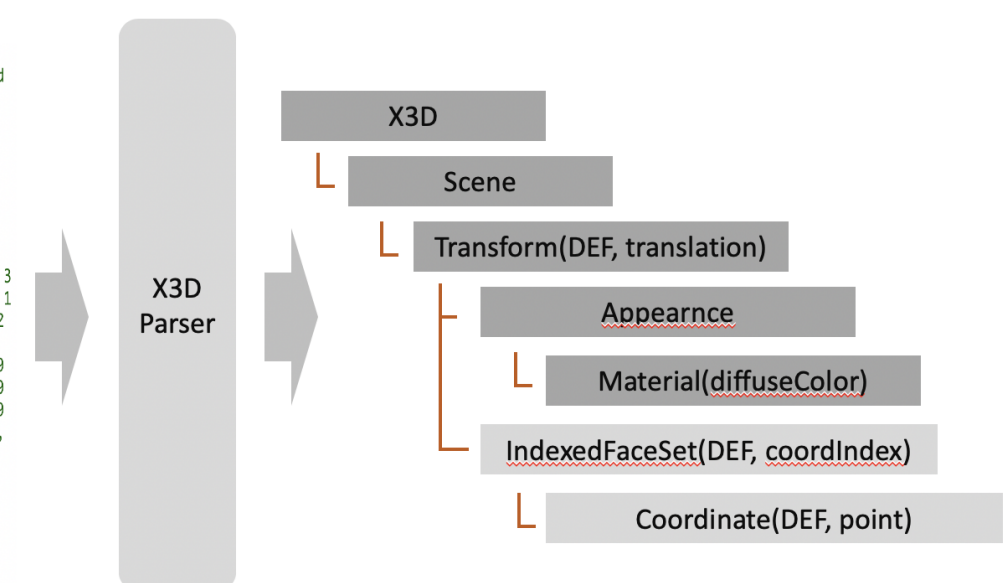

X3D Tree

Figure 3: Parsing X3D nodes
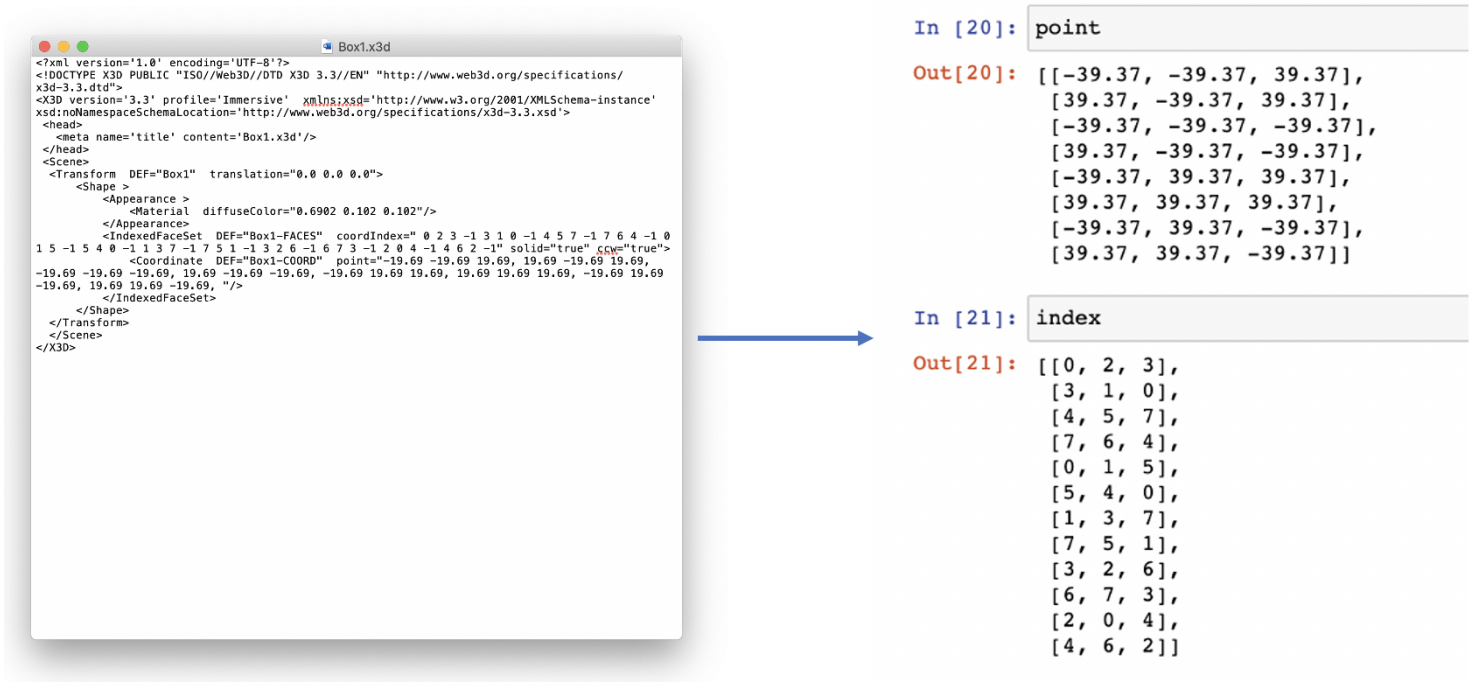

Figure 4: Variables parsed from X3D files

to be trained are located in respective directories. The locations of the objects stored as X3D files are traversed and stored in an array. In this paper, the directories are traversed in consecutive order, and file paths and names are managed as a list. Figure 2 shows the structure of the directories for the data set to be tested for recognition of 3D objects using machine learning. In this experiment, there were seven 3D object types (backpack, book, cap, chair, cup, pen, table) with 100 objects generated per type for testing the algorithm. In total, 700 objects were used for the machine learning.

\subsection{X3D Parsing}

In order to obtain training data from X3D files, an X3D parser has been developed using the Python programming language. Using the parser, CoordIndex and point values are obtained from IndexedFaceSet and Coordinate nodes in the X3D files (Figure 3). The extracted variables of two nodes are mapped to form a new 3 by $\mathrm{N}$ array which consists of faces for an object. The values of CoordIndex and point are stored into lists (Figure 4).

Three index values correspond to a polygon, and point values represent the corresponding coordinates for the polygon. The index and point values are converted to a list structure that can be applied to the machine learning data for DNN. Figure 5 shows the results of converting the index and point values to a list structure that can be used for DNN.

\subsection{D Data Preprocessing}

The number of polygons for each object varies depending on how the object was created and what tools were used. For example, a primitive such as a sphere or a cone can have a different number of 


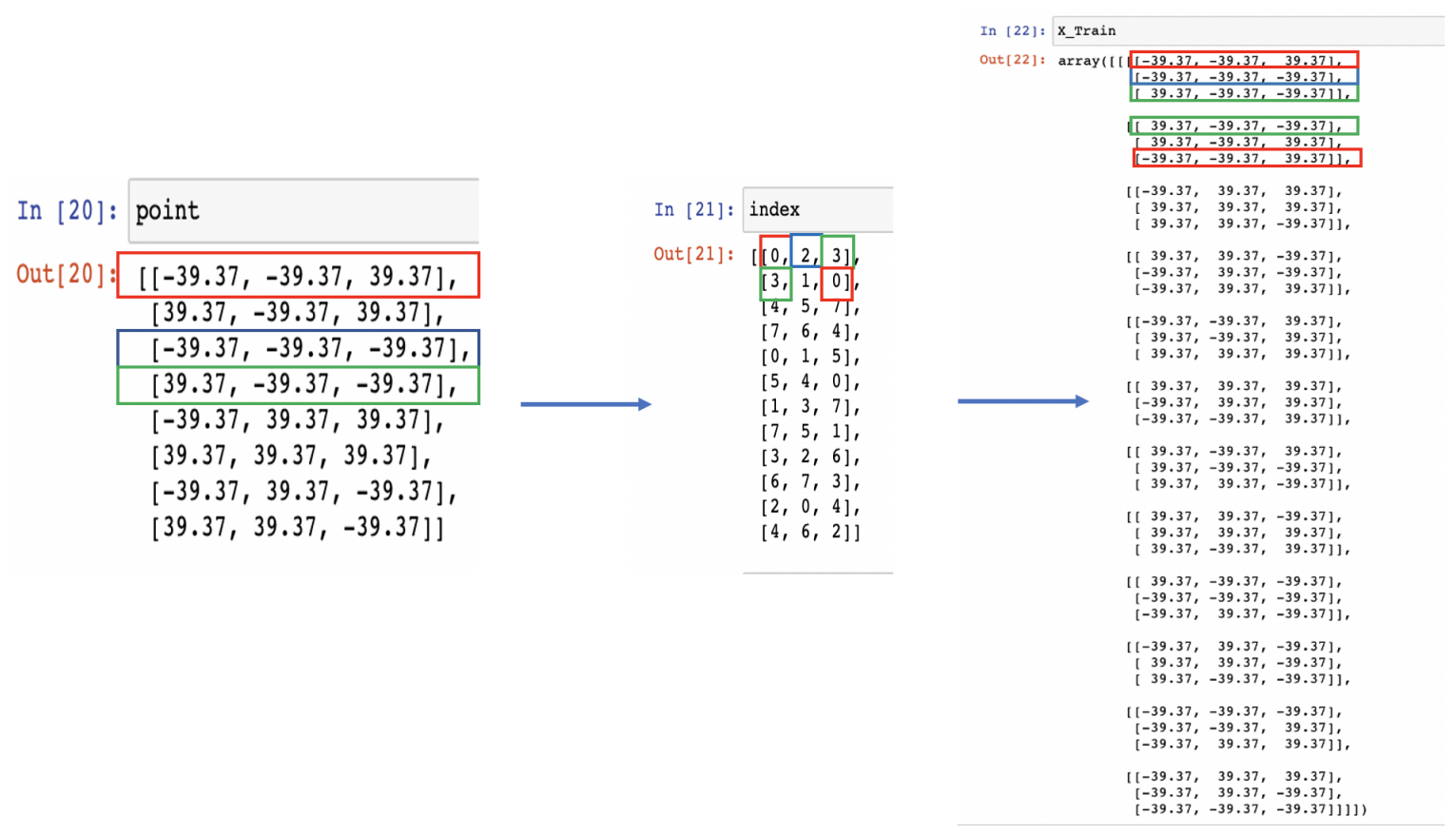

Figure 5: X3D data conversion to a sequential list structure
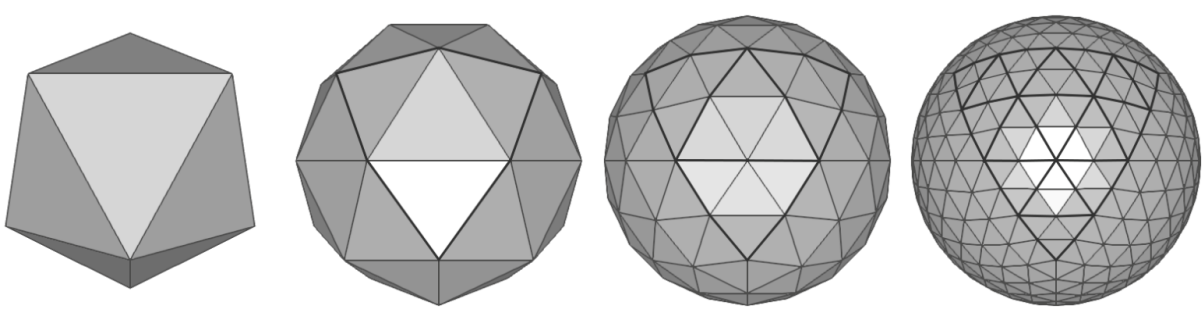

Figure 6: Different numbers of polygons for a sphere

polygons. Figure 6 show this in the case of a sphere. Multiple tens to multiple hundreds of polygons can be used for the primitives.

In this case, if the list of the 3D data values is directly applied for learning using the Keras machine learning algorithm, errors occur because the dimensions of the list of the 3D data don't match. In addition, machine learning can be progressed even if the data includes null values. To avoid this situation, the list of 3D data should be reformatted so as to match the dimensions via data preprocessing. This means that the number of polygons should be matched in the preprocessing (Figure 7). NumPy was used for the preprocessing [van der Walt et al. 2011].

For example, the number of polygons can be set to the larger number of polygons, and then points can be inserted for the point coordinates of the nearest polygon to the smaller number of polygons so that the number of smaller polygons is matched to the larger number of polygons. In Figure 7, vertex1, vertex2, and vertex3 are vertices of the nearest polygon to the last vertex of the 3D object with the smaller number of polygons.

\subsection{Model Initializer}

In this paper, X3D objects were trained using IndexedFaceSet and Coordinate nodes in the objects by deep learning and then a learning model was obtained to test if it can classify and recognize another $\mathrm{X} 3 \mathrm{D}$ object. A sequential model is used for declaring model data for learning and minimum layers are used to avoid overfitting and to have a fast result. In order to receive input 3D data for learning, a dense layer was used. The model is initialized with $(4464,3,3)$ which means 4464 polygons, three vertices and three values for $\mathrm{x}$, $\mathrm{y}$, and $\mathrm{z}$ per polygon (Figure 8).

These data were converted from a three-dimensional array to a one-dimensional array using a flattened layer. In addition, three more dense layers were used for distributing the seven sets of the 3D model data. Figure 8 shows the summary of the model for learning using the sets of $3 \mathrm{D}$ data.

\subsection{Machine Learning Results and Evaluation}

Seven sets of 3D data (100 backpacks, 100 books, 100 caps, 100 chairs, 100 cups, 100 pens, 100 tables) were used for the machine 


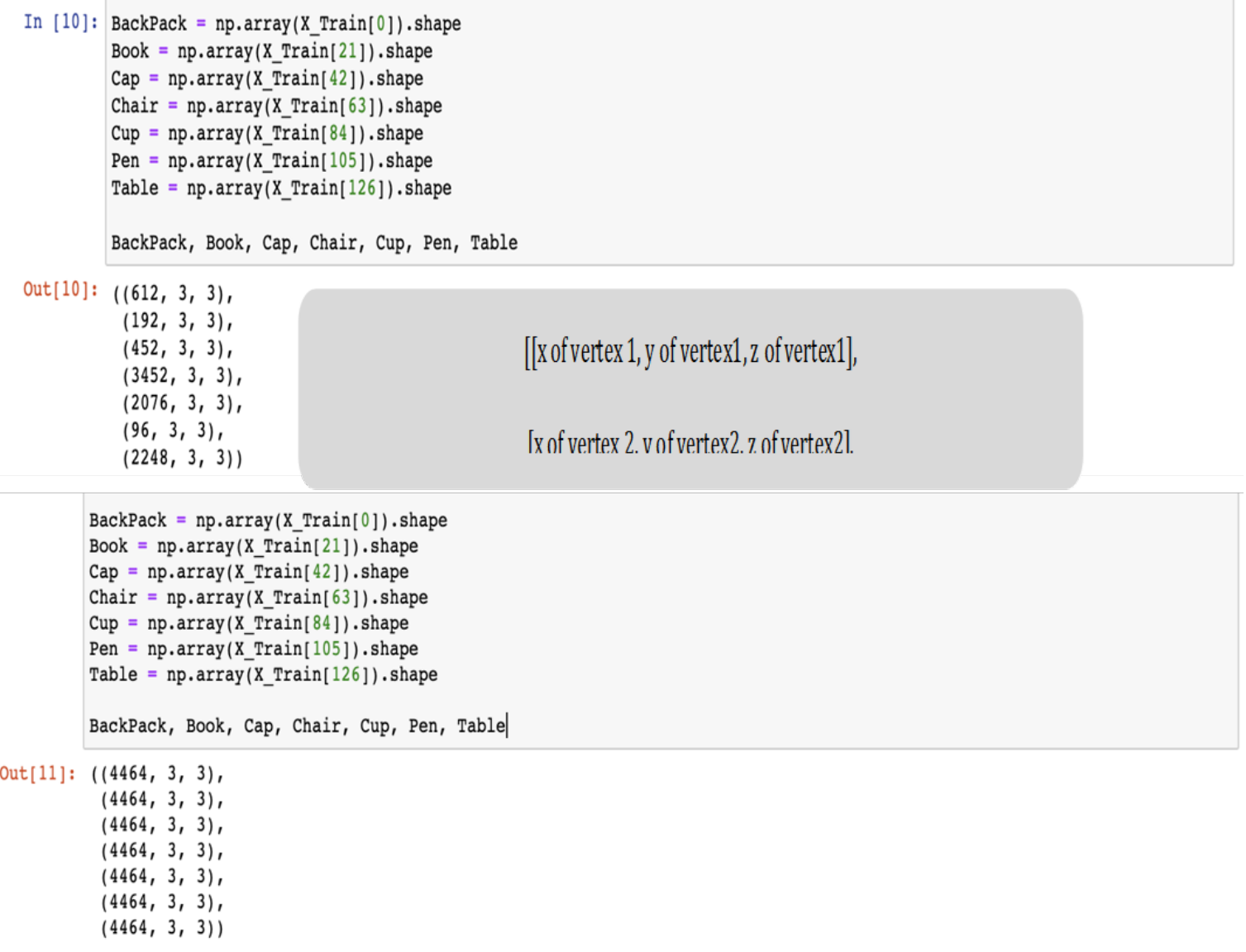

Figure 7: Preprocessing for matching the number of polygons

Model: "sequential_1"

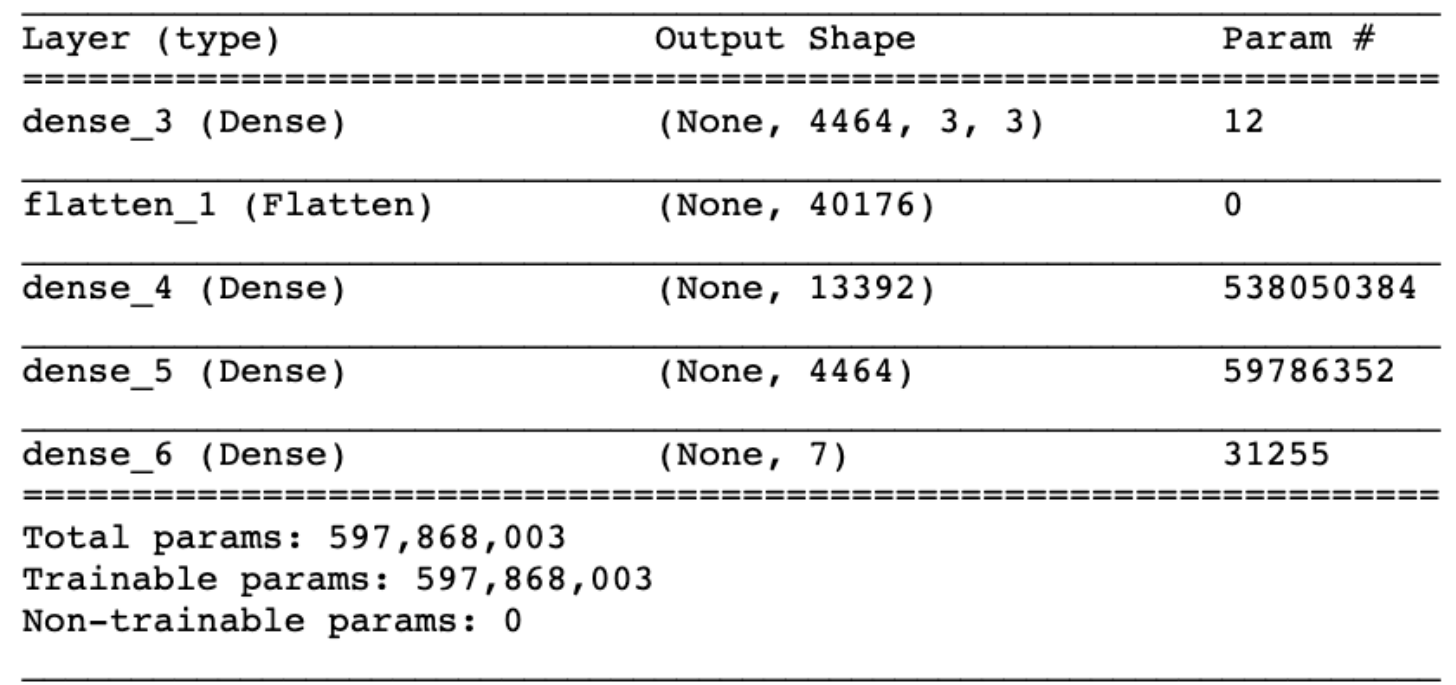

Figure 8: Model initializer for learning 


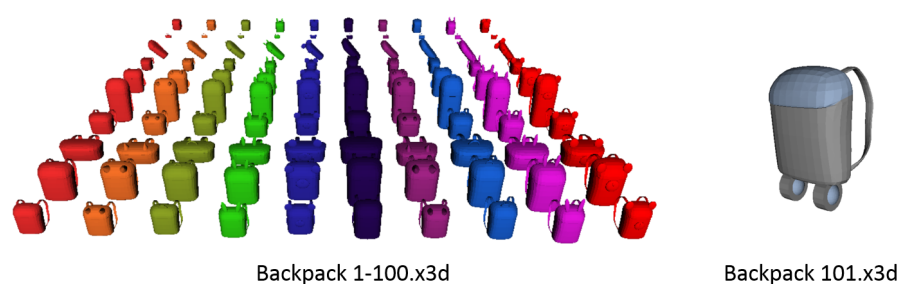

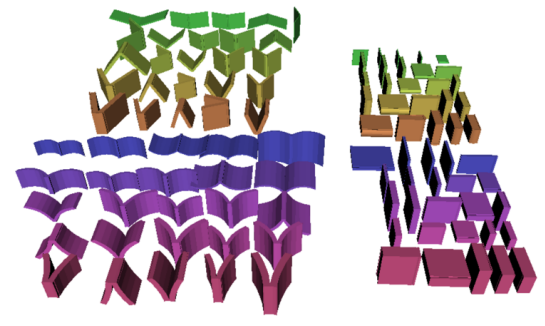

Book 1-100.x3d

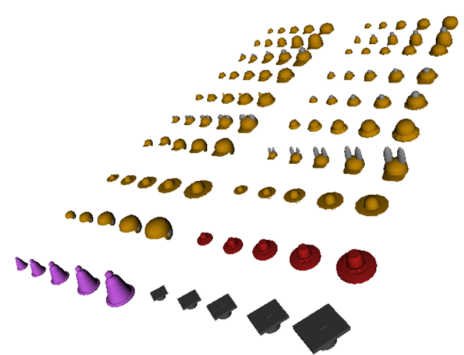

Cap 1-100.x3d

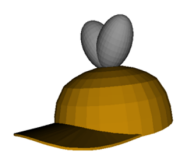

Cap101.x3d

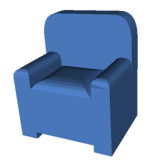

Chair 101.x3d

Chair 1-100.x3d

Book 101.x3d
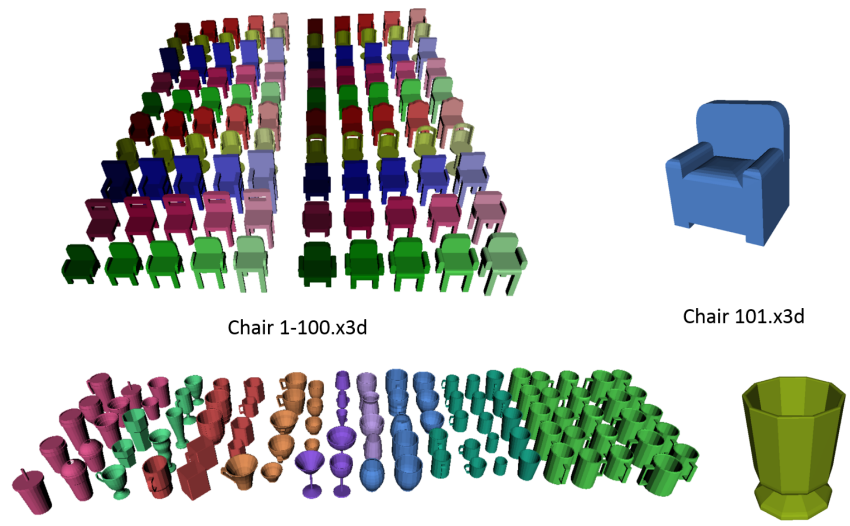

Cup 1-100.x3d

Cup 101.x3d
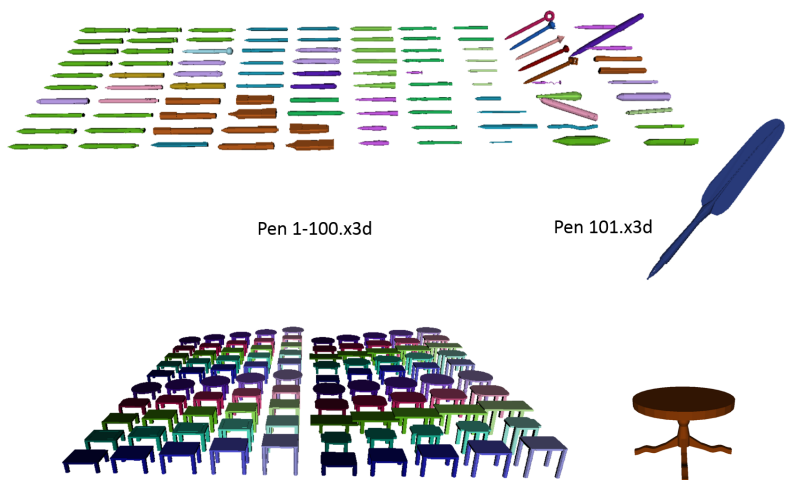

Table 1-100.x3d

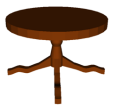

Table 101.x3d

Figure 9: X3D Models used for learning (1-100) and estimation (101) 
Table 1: Loss values and accuracy for each test

\begin{tabular}{llllll}
\hline $\begin{array}{l}\text { Number of 3D models } \\
\text { used }\end{array}$ & 20 & 40 & 60 & 80 & 100 \\
\hline Loss value & 159.18 & 28.96 & 1.36 & 0.28 & 1.22 \\
Accuracy & $57.14 \%$ & $71.43 \%$ & $71.43 \%$ & $100 \%$ & $85 \%$ \\
\hline
\end{tabular}

Table 2: Evaluation results of recognizing 3D objects according to the number of models

\begin{tabular}{llllll}
\hline $\begin{array}{l}\text { 3D model type / number } \\
\text { of 3D models used }\end{array}$ & 20 & 40 & 60 & 80 & 100 \\
\hline Backpack & $0 \%$ & $99 \%$ & $1 \%$ & $99 \%$ & $0 \%$ \\
Book & $100 \%$ & $100 \%$ & $100 \%$ & $100 \%$ & $100 \%$ \\
Cap & $0 \%$ & $0 \%$ & $100 \%$ & $100 \%$ & $100 \%$ \\
Chair & $100 \%$ & $0 \%$ & $0 \%$ & $100 \%$ & $100 \%$ \\
Cup & $100 \%$ & $100 \%$ & $100 \%$ & $100 \%$ & $100 \%$ \\
Pen & $100 \%$ & $100 \%$ & $3 \%$ & $100 \%$ & $100 \%$ \\
Table & $0 \%$ & $100 \%$ & $3 \%$ & $100 \%$ & $100 \%$ \\
\hline
\end{tabular}

learning. These data sets were used to obtain a learning model using $20,40,60,80$, and 100 objects. This means that the learning model was obtained using all the sets of 3D data. In order to recognize a $3 \mathrm{D}$ model, an additional $3 \mathrm{D}$ model for each primitive type $\left(101^{\text {st }}\right.$ backpack, $101^{\text {st }}$ book, $101^{\text {st }}$ cap, $101^{\text {st }}$ chair, $101^{\text {st }}$ cup, $101^{\text {st }}$ pen, and $101^{\text {st }}$ table) is applied to the learning model and an estimate is made of how much it is similar to the learning model. A loss function of Categorical Cross-Entropy Loss was used to estimate the accuracy [2]. Table 1 shows loss values and accuracy for each test. For loss values, a value nearest to zero indicates the greatest accuracy. For each 3D model type, an additional 3D model was estimated, as in Table 2. The numbers represent how much each of the additional 3D models is similar to the learning model. Figure 9 shows the 3D models used for learning and estimation in this paper. In order to obtain a learning model, 100 X3D models for learning and one additional X3D model for estimation were created for each type. In total, $700 \mathrm{X} 3 \mathrm{D}$ models for learning, and 7 additional X3D models for estimation were generated and used.

Figure 10 shows the evaluation results of recognizing the chair and table models in the case of learning using 80 3D models. It shows that the learning model corresponds to approximately $100 \%$ recognition for the table and the chair at over 80 X3D models. According to the results, the case where 20 data sets were used for estimation had the lowest recognition. This is due to insufficient learning with a small number of 3D data sets. The case of using $803 \mathrm{D}$ models was the most accurate. At 100 models, accuracy decreased and loss values increased. It is assumed that that the parameters used for learning increased and therefore accuracy decreased with the increase of test 3D data sets.
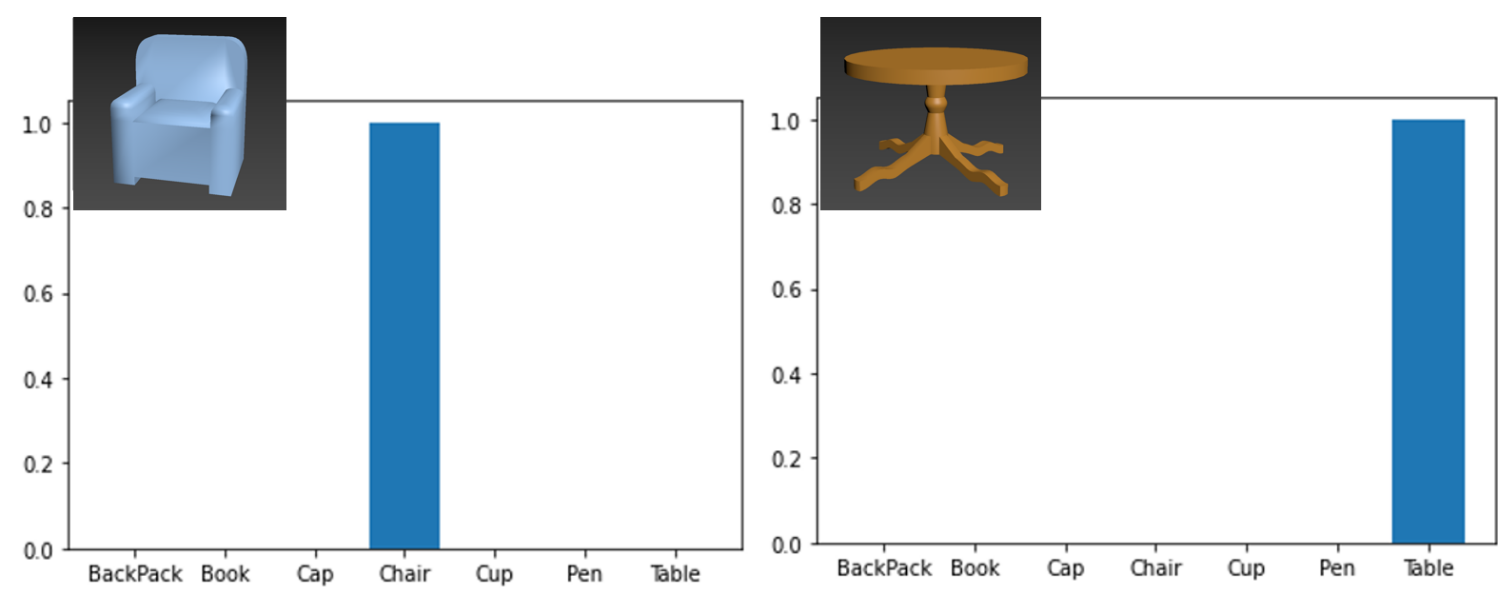

Figure 10: Evaluation results of recognizing the table and the chair models 


\section{CONCLUSIONS}

In this paper, a 3D object recognition method using X3D and a machine learning algorithm based on Keras and the Python programming language was described. Learning models were obtained by applying the machine learning algorithm to 700 X3D data models with seven different model types (each model type had 100 X3D models). An additional X3D model for each model type was used to evaluate the accuracy of recognizing an X3D object using the machine learning model. Two aspects were analyzed: One is that the DNN algorithm using geometric coordinates of 3D models provides more accurate recognition. The other is that the nearest vertices should be padded when the objects to be compared have different numbers of polygons for the machine learning algorithm. This can result in better accuracy than zero padding used in other research.

Based on the experimentation results of this paper, X3D is suitable for recognizing 3D objects with machine learning. As X3D databases grow, it is expected that X3D can be used in big data industry to provide many valuable functions such as 3D object search, retrieval, and storage. Further study includes experimentation around the recognition of more complex objects and enhancing the functions of preprocessing to reduce loss values. In addition, object properties including color, texture, location and orientation for 3D object recognition should also be considered for future work.

\section{ACKNOWLEDGMENTS}

This work was supported by the Standard Technology Development and Spread Program of KATS/KEIT (10085589), and by MSIT, Korea, under the ITRC (Information Technology Research Center) support program (IITP-2020-2016-0-00312) supervised by the IITP.

\section{REFERENCES}

James G. Shanahan and Liang Dai. 2019. "Realtime Object Detection via Deep Learningbased Pipelines”, CIKM'19: Proceedings of the 28th ACM International Conference on Information and Knowedge.

Jonggi Hong, Kyungjun Lee, June Xu, and Hernisa Kacorri. 2019. "Exploring Machine Teaching for Object Recognition with the Crowd", CHI EA'19: Extended Abstracts of the 2019 CHI Conference on Human Factors in Computing Systems.

Xinyi Zhou, Wei Gong, WenLong Fu, and Fengtong Du. 2017. "Application of deep learning in object detection", ICIS 2017, Volume 1, pp. 631-634.

Kaiming He, Xiangyu Zhang, Shaoqing Ren, and Jian Sun. December 2015. "Deep Residual Learning for Image Recognition", arXiv.

Xingchao Peng, Baochen Sun, Karim Ali, and Kate Saenko. 2015. "Learning Deep Object Detectors from 3D Models", ICCV 2015, Volume 1, pp. 1278-1286.

S. Zhi, Y. Liu, X. Li, and Y. Guo. 2017. "LightNet: A Lightweight 3D Convolutional Neural Network for Real-Time 3D Object Recognition”, Eurographics Proceedings. Yuqi Zhang, Yongzhen Huang, and Liang Wang. 2017. "Multi-task Deep Learning for Fast Online Multiple Object Tracking”, ACPR 2017, Volume 1, pp. 138-143.

M. A. Rodrigues and A. Robinson. 2011. "Real-time 3D face recognition using line projection and mesh sampling”, 3DOR'11: Proceedings of the 4th Eurographics conference on 3D Object Retrieval, pp. 9-16.

Stefano Berretti, Mohamed Daoudi, Pavan Turaga, and Anup Basu. 2018. "Representation, Analysis, and Recognition of 3D Humans: A Survey", ACM Transactions on Multimedia Computing, Communications, and Applications, Volume 14, Issue 1s, pp.1-36.

Zhilu Zhang and Mert R. Sabuncu. 2018. "Generalized Cross Entrophy Loss for Training Deep Neural Networks with Noizy Labels", arXiv.

Yaoshiang Ho and Samuel Wookey. 2010. "The Real-World-Weight Cross-Etrophy Loss function: Modeling the Costs of Mislabeling", IEEE Access, Vol. 8, pp. 4806-4813.

Sebastian Rudger. 2017. "An overview of gradient descent optimization algorithms", arXiv.

Diederik P. Kingma. 2015. “ADAM: A Method for Stochastic Optimization”, Proceedings of ICLR.

Mahesh Chandra Mukkamala and Matthias Hein. August 2017. "Variants of RMSProp and Adagrad with logarithmic regret bounds", ICML'17: Proceedings of the 34th International Conference on Machine Learning, Vol. 70, pp. 2545-2553.

ISO/IEC 19775-1: 2013 Extensible 3D (X3D). 2013.

Stefan van der Walt, S. Chris Colbert, and Gael Varoquaux. 2011. "The NumPy array: a structure for efficient numerical calculation”, IEEE Computing in science and engineering. 